\title{
Nueva estela discoidea en Sepúlveda, Segovia (España). A propósito de una recensión a $E R S g$
}

\author{
Juan SANTOS YanguAS \\ Universidad del País Vasco \\ juan.santos@ehu.es \\ Ángel Luis Hoces de la Guardia Bermejo \\ Investigador Independiente. Segovia \\ hdg@hdgsg.net
}

\section{RESUMEN}

En este artículo contestamos a una recensión a ERSg publicada en 2005, en la que el Dr. D. Manuel Ramírez Sánchez daba a conocer una pieza epigráfica en la plaza delante de la iglesia de la Virgen de la Peña, la cual no pudimos ver, pues, entre su inspección y la nuestra, hubo obras de remodelación del pavimento, por lo que la pieza quedó oculta. Por otra parte, comparando la pieza en sus fotos pudimos comprobar que no es romana, como mostramos en nuestro comentario.

Palabras clave: Epigrafía, Edad Media, estrella, estela funeraria.

\section{A New Discoid Stele in Sepúlveda (Segovia). About a Recension to ERSG}

\begin{abstract}
In this article we answer to a recession ERSg published in 2005, in which Dr. D. Manuel Ramírez Sánchez made known epigraphic piece in the square in front of the church of Virgen de la Peña, which we could not see, then, between inspection and ours, there was remodeling the pavement, so that the piece was hidden. Moreover, comparing the piece in the photos could prove is not Roman, as shown in our reviews.
\end{abstract}

Key words: Epigraphy, Middle Ages, Star, Gravestone. 
Por una amable comunicación del Dr. Ramírez Sánchez ${ }^{1}$ tuvimos conocimiento de la existencia de una lápida con una estrella de seis puntas o "una rosácea sexapétala inscrita en un doble círculo de $30 \mathrm{~cm}$ de diámetro" en la iglesia de la Virgen de la Peña, en la localidad de Sepúlveda (Segovia), situada en la horquilla de los ríos Duratón y Caslilla, en cuyo núcleo urbano y alrededores ya se tenía conocimiento de una decena de inscripciones de época romana, algunas de ellas rupestres, la más importante de las cuales la que se halla en la orilla de río Duratón, cerca del Puente Talcano, y varias en las paredes internas de cuevas naturales, generalmente acondicionadas, en la margen izquierda del río Caslilla².

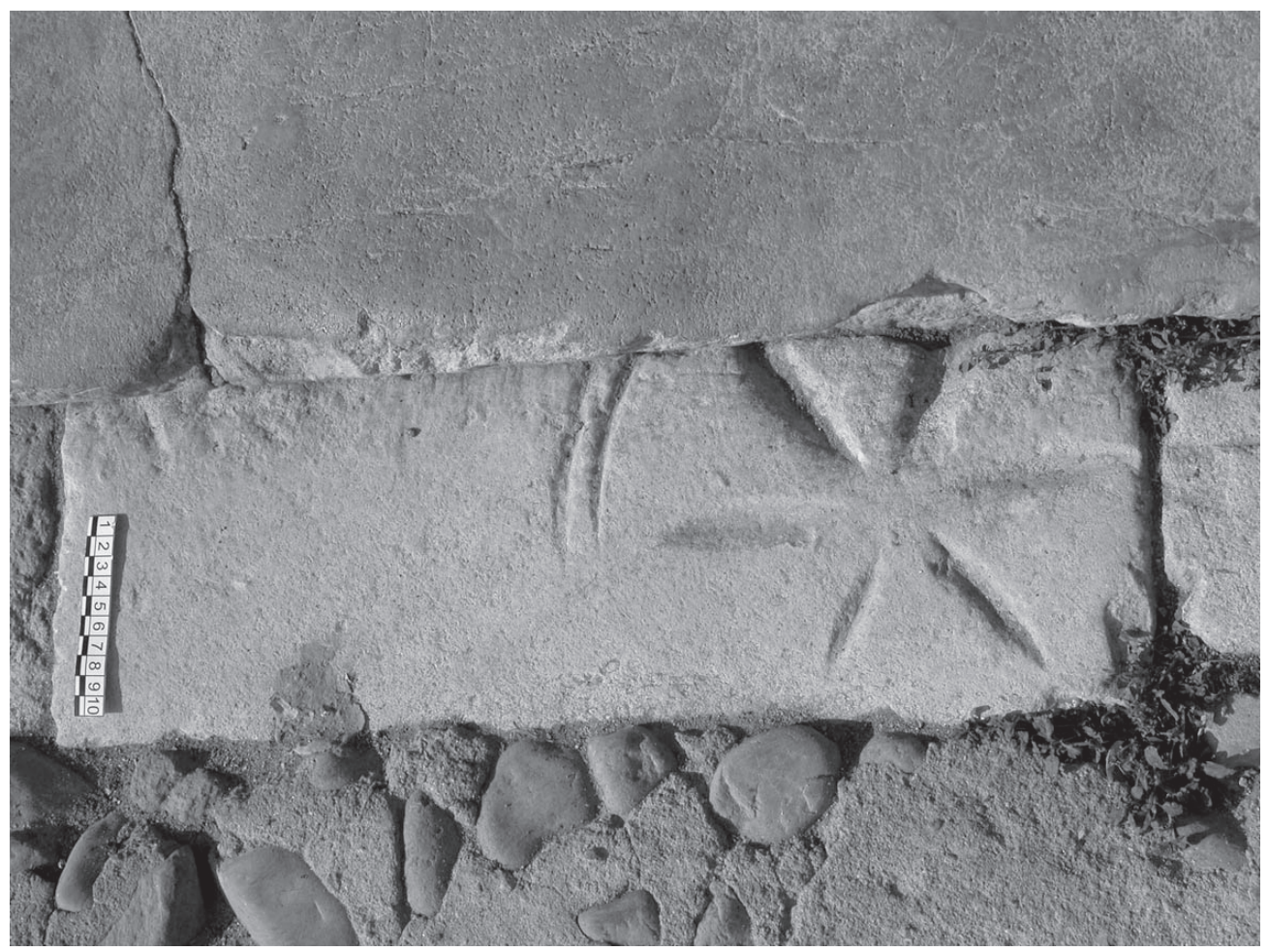

Fig. 1: Lápida con estrella de seis brazos (Ramírez).

A partir de la foto que amablemente nos envió el propio Ramírez (fig.1), de la cual se deducía que estaba en el exterior de la iglesia, indagamos sobre su localización los días 12.10.2006 y 1.11.2006. D. Pedro Manuel Fernández, vecino de Sepúlveda, nos

1 Extremo reflejado por Ramírez en su recensión a nuestra Epigrafía romana de Segovia y su provincia, en Gerion 24.2 (2006): 136-140. Le agradecemos su comunicación de la existencia de la pieza, antes incluso de ofrecer él la noticia en la reseña indicada.

2 J. Santos Yanguas, A.L. Hoces de la Guardia Bermejo y J. del Hoyo, Epigrafía romana de Segovia y su provincia, Segovia, 2005, 231-240, con una pequeña introducción a la ubicación geográfica de esta población y al lugar en el que se han hallado las distintas inscripciones. 


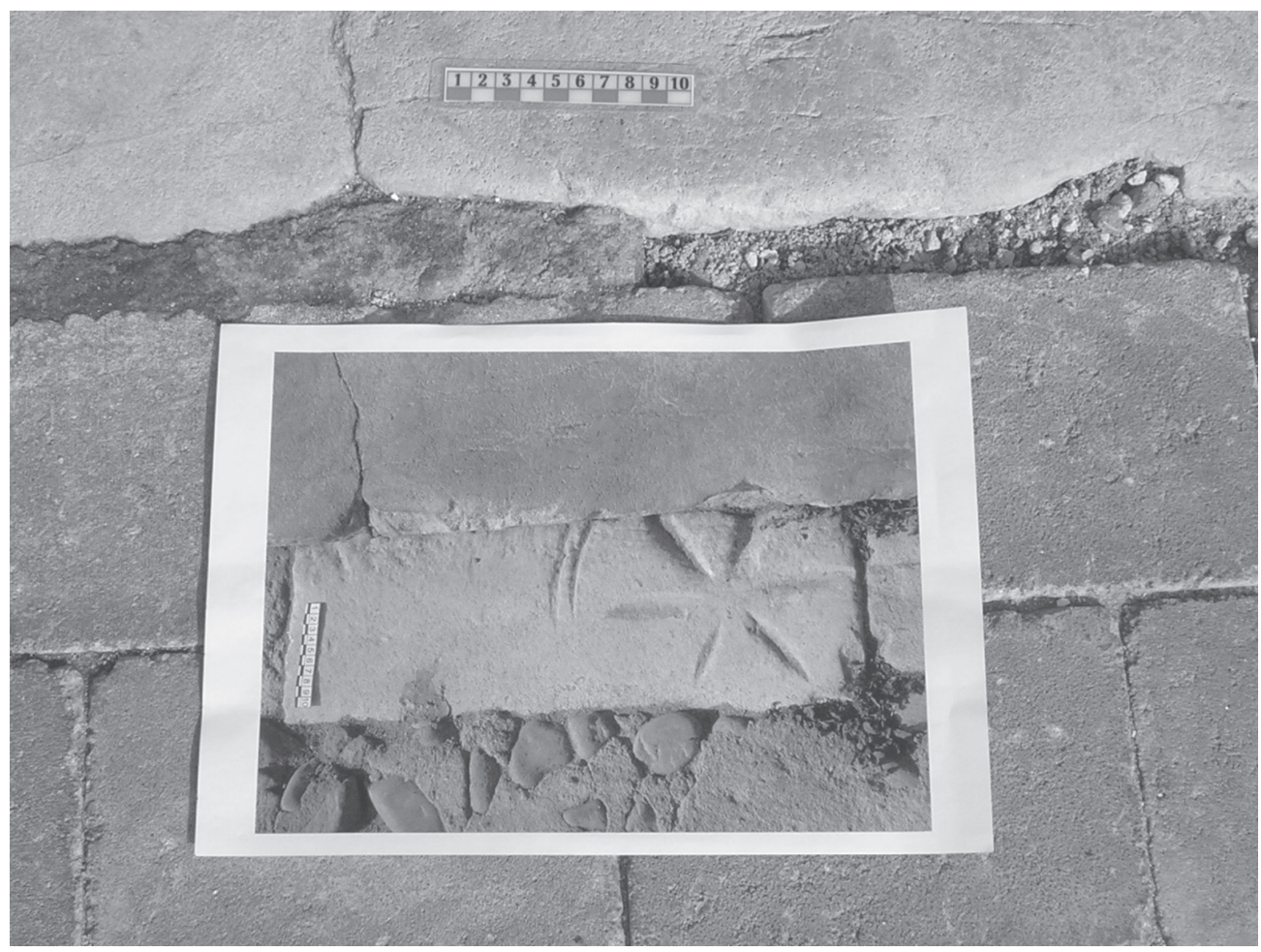

Fig. 2: Posible localización actual.

indicó que se hallaba en el exterior de la iglesia de la Virgen de la Peña, en el suelo, justo por delante del primer escalón de acceso al pórtico, desconociéndose su procedencia original. Asimismo el Sr. Fernández nos trasmitió la noticia de que durante el verano de 2006 se habían realizado obras de arreglo del pavimento de toda la zona afectando también al suelo de la plaza a la que se abre el pórtico de la iglesia. Según su información estas obras se realizaron sin remoción de la pieza, quedando ésta tapada por el nuevo pavimento (fig. 2) ${ }^{3}$. Sin embargo, a partir del análisis de la fotografía que nos envió el Dr. Ramírez y del análisis de la pavimentación actual de la plaza, se deduce que el nivel en el que estaba situada la estela ha tenido que ser rebajado, para lo cual ésta ha debido ser extraída de su ubicación o rehundida (fig. 3). En el primer caso, ¿dónde se encuentra actualmente la pieza?

Según descripción del propio Ramírez, "se trata de un fragmento de estela caliza, de $(63) \times(18) \mathrm{cm}$, que no conserva texto alguno, en cuya parte superior se conserva una rosácea sexapétala inscrita en un doble círculo de $30 \mathrm{~cm}$ de diámetro".

Es posible que se trate de una estela discoidea de arenisca blanquecina, recortada en todos sus lados. Presenta una estrella hexapétala $(30 \mathrm{~cm} \varnothing)$ enmarcada por doble círculo de 30 y $34 \mathrm{~cm} \varnothing$ inscrito.

\footnotetext{
${ }^{3}$ Queremos agradecer de nuevo a D. Pedro Manuel Fernández los datos ofrecidos referentes a las obras realizadas en la zona.
} 


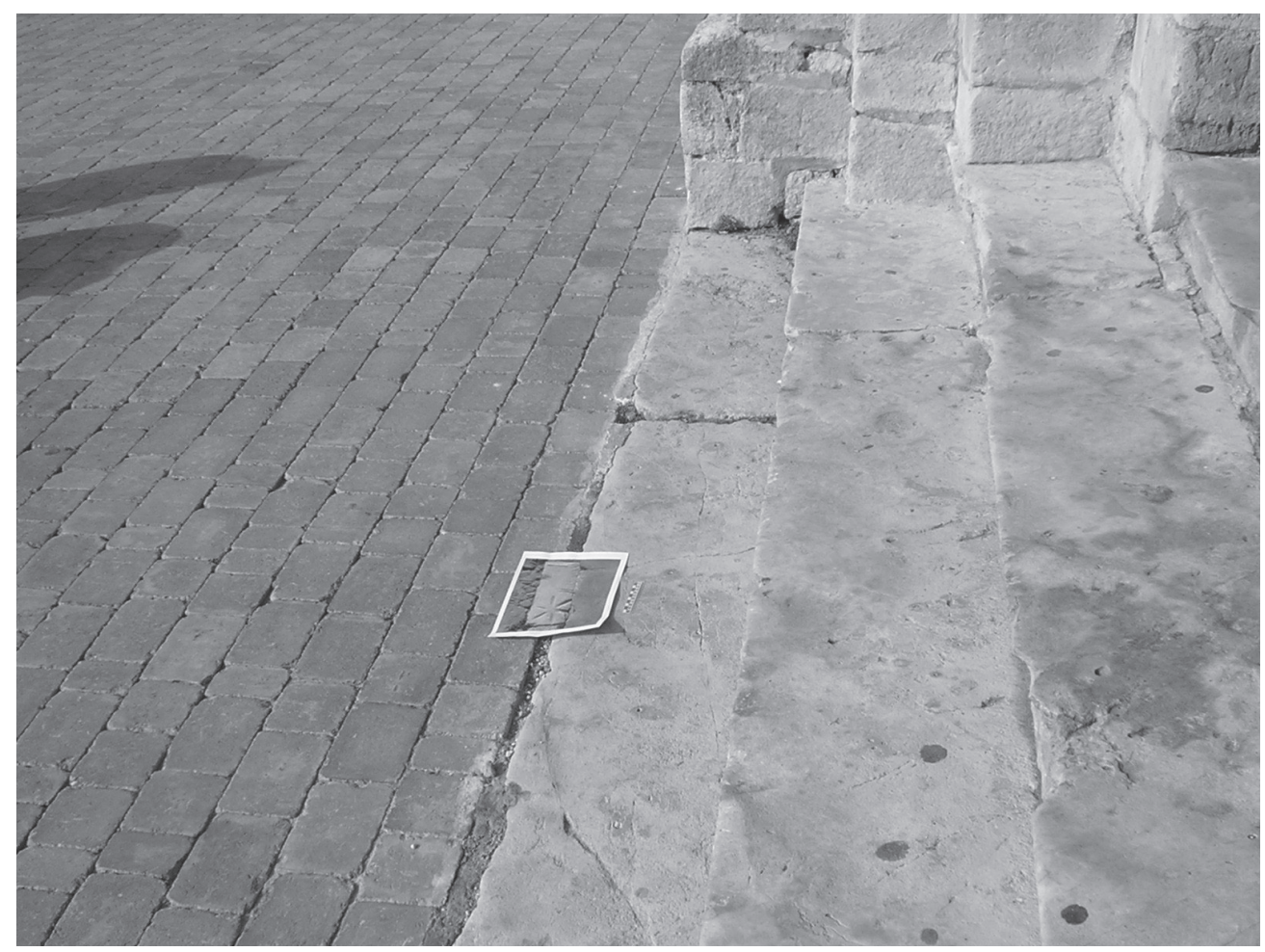

Fig. 3: Pavimento actual.

Por otra parte, no parece que esta pieza, de la que no teníamos noticia cuando realizamos el corpus de la provincia de Segovia, deba ser considerada entre las de época romana, pues se asemeja más a estelas medievales ya conocidas, por ejemplo en Sagunto, Valencia (De la Casa y Monraval 1994: 499, n 1-R y 6-R; 501, n 20-C y n $\left.{ }^{\text {o } 22-R ; ~ y ~ 503, ~ n o ~ 34-R ~}\right)$ y Portugal (Beleza 1994: 690, no 39b).

De acuerdo con todos estos datos, la reconstrucción ideal de la pieza es la que proponemos en la fig. 4.

${ }^{4}$ En el artículo de De la Casa y Manraval, si bien hay descripción de piezas y dibujo de las mismas, éstos y ésas no se corresponden por el número asignado. Todas las piezas son discoideas. Así, tomamos la descripción y numeración de los propios dibujos: Dibujo 1: decoración anverso: cruz griega inscrita en un círculo; decoración reverso: flor de 6 pétalos. Dibujo 6: decoración anverso: cruz griega de brazos rectos inscrita en un círculo; decoración reverso: flor de 6 pétalos. Dibujo 20: decoración anverso: flor de 6 pétalos; decoración reverso: cruz griega inscrita en un círculo. Dibujo 22: decoración anverso: cruz griega inscrita en un círculo; decoración reverso: flor de 6 pétalos. Dibujo 34: decoración anverso: cruz griega inscrita en un círculo; decoración reverso: flor de 6 pétalos. 


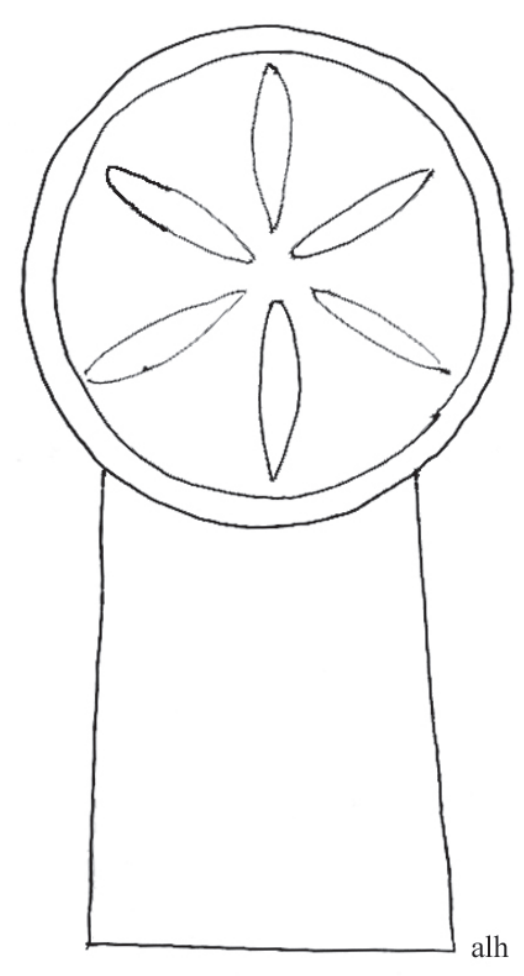

Fig. 4: Reconstrucción ideal.

\section{Bibliografía}

Beleza Moreira, J. (1994): "Mais alhunas profissoes representadas en estelas discóides portuguesas", en Actas del V Congreso Internacional de Estelas Funerarias. Soria, del 28 de abril al 1 de mayo de 1993 (ed. C. de la Casa), Soria, 1994, 663693.

DE la CASA, C. y Monraval, M. (1994): "Conjunto de estelas funerarias medievales de Sagunto (Valencia)", en Actas del V Congreso Internacional de Estelas Funerarias.Soria, del 28 de abril al 1 de mayo de 1993 (ed. C. de la Casa), Soria, 1994, 497-507. 